\title{
Scattering of Light and Pulling Optical Forces for Hyperbolic-Metamaterial Spheres
}

\author{
Novitsky, Andrey; Zhukovsky, Sergei; Lavrinenko, Andrei
}

\section{Published in:}

Proceedings of 8th International Congress on Advanced Electromagnetic Materials in Microwaves and Optics (METAMATERIALS 2014)

Link to article, DOI:

10.1109/MetaMaterials.2014.6948655

Publication date:

2014

Document Version

Peer reviewed version

Link back to DTU Orbit

Citation (APA):

Novitsky, A., Zhukovsky, S., \& Lavrinenko, A. (2014). Scattering of Light and Pulling Optical Forces for Hyperbolic-Metamaterial Spheres. In Proceedings of 8th International Congress on Advanced Electromagnetic Materials in Microwaves and Optics (METAMATERIALS 2014) (pp. 226-228). IEEE. https://doi.org/10.1109/MetaMaterials.2014.6948655

\section{General rights}

Copyright and moral rights for the publications made accessible in the public portal are retained by the authors and/or other copyright owners and it is a condition of accessing publications that users recognise and abide by the legal requirements associated with these rights.

- Users may download and print one copy of any publication from the public portal for the purpose of private study or research.

- You may not further distribute the material or use it for any profit-making activity or commercial gain

- You may freely distribute the URL identifying the publication in the public portal 


\title{
Scattering of Light and Pulling Optical Forces for Hyperbolic-Metamaterial Spheres
}

\author{
A.V. Novitsky ${ }^{1}$, S.V. Zhukovsky ${ }^{2}$, and A.V. Lavrinenko ${ }^{2}$ \\ ${ }^{1}$ Belarusian State University, Department of Theoretical Physics and Astrophysics, \\ Nezavisimosti Ave. 4, 220030, Minsk, Belarus \\ ${ }^{2}$ DTU Fotonik, Technical University of Denmark, Ørsteds Plads 343, DK-2800, Kgs. Lyngby, Denmark \\ andrey.novitsky@tut.by
}

\begin{abstract}
We discuss electromagnetic fields and optical forces acting on microspheres made of extremely anisotropic medium. We propose an energy-based criterion for choosing an appropriate solution of Maxwell's equations inside the sphere when two solutions are singular at the center. We investigate the material parameters of a lossy hyperbolic-metamaterial sphere that can be pulled by a nonparaxial, gradientless Bessel beam.
\end{abstract}

\section{INTRODUCTION}

Light-matter interaction in classical physics can be described using the Mie scattering theory [1]. The scattered electromagnetic field is employed for calculating optical forces acting on a micro-object. These forces stem from the excitation of multipole moments in the object. For example, the dynamics of small particles is known to be controlled solely by the dipole moments [2]. Generally, an optical force can be divided into a gradient force, caused by the intensity gradient and employed in conventional optical tweezers, and a non-conservative force, which exists in homogeneous fields and can be called the radiation-pressure force. It was shown previously that the "pressure" can be negative: the non-conservative force can attract an isotropic spherical particle towards the light source owing to the interaction of multipoles excited by a non-paraxial light beam [3-6]. For example, particles can be attracted by a non-diffracting Bessel beam in which there is no intensity gradient in the direction of propagation, i.e. the gradient force equals zero in this direction $[3,4]$.

In this paper we study the pulling forces acting on anisotropic spheres. The most interesting case includes microspheres made of a hyperbolic metamaterial (HMM) [7]. It is expected that strong enhancement of the photonic density of states in HMMs, which opens up numerous potential applications in subwavelength imaging, lithography, and quantum photonics, would also result in strong modification of the pulling force effect for HMM spheres.

\section{Spherical Waves in Indefinite Media: Energy-BASEd CRiterion}

Let a nonmagnetic spherical particle of radius $R$ be characterized by a dielectric permittivity tensor

$$
\varepsilon=\varepsilon_{r} \mathbf{e}_{r} \otimes \mathbf{e}_{r}+\varepsilon_{t}\left(\mathbf{e}_{\theta} \otimes \mathbf{e}_{\theta}+\mathbf{e}_{\varphi} \otimes \mathbf{e}_{\varphi}\right),
$$

where $\mathbf{e}_{r}, \mathbf{e}_{\theta}$, and $\mathbf{e}_{\varphi}$ are the basis vectors in spherical coordinates, $(\mathbf{a} \otimes \mathbf{b})_{i j}=a_{i} b_{j}$ is a dyad. Radial $\varepsilon_{r}=\varepsilon_{r}^{\prime}+i \varepsilon_{r}^{\prime \prime}$ and transverse $\varepsilon_{t}=\varepsilon_{t}^{\prime}+i \varepsilon_{t}^{\prime \prime}$ permittivities are complex numbers. When $\varepsilon_{t}^{\prime} \varepsilon_{r}^{\prime}<0$, the anisotropic medium is called indefinite, and can be realized in hyperbolic metamaterials [7].

An incident monochromatic electromagnetic wave with angular frequency $\omega$ induces electromagnetic field inside the spherical particle. Bessel functions at small arguments (near the center of the sphere $r=0$ ) can be approximated by power-law functions, therefore, the electric field of two independent solutions with the smallest angular momentum number $l=1$ is estimated as

$$
E^{(1)} \sim \frac{j_{\nu-1 / 2}\left(\sqrt{\varepsilon_{t}} k_{0} r\right)}{r} \sim r^{\nu-\frac{3}{2}}, \quad E^{(2)} \sim \frac{y_{\nu-1 / 2}\left(\sqrt{\varepsilon_{t}} k_{0} r\right)}{r} \sim r^{-\nu-\frac{3}{2}}, \quad \text { where } \nu=\sqrt{l(l+1) \frac{\varepsilon_{t}}{\varepsilon_{r}}+\frac{1}{4}},
$$

$j_{m}$ and $y_{m}$ are the spherical Bessel function of the first and second kind, respectively, and $k_{0}$ is the wavenumber in vacuum. We notice that for $\operatorname{Re}(\nu)<3 / 2$ the electric fields for both solutions $E^{(1)}$ and $E^{(2)}$ at the center 
of a spherical particle become infinite. In the case of a transparent medium with real $\varepsilon_{t}$ and $\varepsilon_{r}$, the condition $\operatorname{Re}(\nu)=\nu=\sqrt{2 \varepsilon_{t} / \varepsilon_{r}+1 / 4}<3 / 2$ holds true for anisotropic materials with $\varepsilon_{t} / \varepsilon_{r}<1$. This includes all hyperbolic metamaterials, which are characterized by $\varepsilon_{t} \varepsilon_{r}<0$.

Since both solutions of the Maxwell equations are singular at the center, we need a criterion for choosing the appropriate one. We claim that the physically correct solution should comply with the finiteness of the electromagnetic energy stored inside the sphere:

$$
\int_{\text {sphere }}|\mathbf{E}|^{2} d V<\infty
$$

By substituting the first solution in (2) into Eq. (3) we derive

$$
\int_{0}^{R}\left|E^{(1)}\right|^{2} r^{2} d r \sim \int_{0}^{R} r^{2 \operatorname{Re}(\nu)-1} d r
$$

If $\operatorname{Re}(\nu)>0$, the integral always results in the finite energy inside the sphere. If $\operatorname{Re}(\nu)<0$, we should choose the second solution $E^{(2)}$. The intermediate case $\operatorname{Re}(\nu)=0$ corresponds to the anisotropic medium with $\operatorname{Im}\left(\varepsilon_{t} / \varepsilon_{r}\right)=$ 0 and $\operatorname{Re}\left(\varepsilon_{t} / \varepsilon_{r}\right)+1 / 8<0$ and results in the logarithmically divergent integral. However, $\operatorname{Re}(\nu)=0$ is a very restrictive condition that cannot be realized in practice. For example, lossless medium is an idealization; realistic passive transparent media have the permittivities $\varepsilon_{t}+\mathrm{i} \delta_{t}$ and $\varepsilon_{r}+\mathrm{i} \delta_{r}$, where $\delta_{t, r}>0$ is a small quantity. Thus, the appropriate solutions inside anisotropic spherical particles should ensure finite energy according to Eq. (3).

\section{Pulling Forces For Hyperbolic-Metamaterial Spheres}

We consider a spherical particle (bead) in the dipole approximation. Its electric and magnetic polarizabilities are expressed in terms of the Mie coefficients $a_{1}$ and $b_{1}$ as

$$
\alpha_{e}=\frac{3 \mathrm{i} a_{1}}{2 k_{0}^{3}}, \quad \alpha_{m}=\frac{3 \mathrm{i} b_{1}}{2 k_{0}^{3}} ; \quad \alpha_{e, m}=\frac{\alpha_{e, m}^{(0)} / k_{0}^{3}}{1-2 \mathrm{i} \alpha_{e, m}^{(0)} / 3}, \quad \alpha_{e}^{(0)}=\frac{\varepsilon_{2}-\nu / 2-1 / 4}{\varepsilon_{2}+\nu+1 / 2}\left(k_{0} R\right)^{3}, \quad \alpha_{m}^{(0)}=0 .
$$

The last expression for $\alpha_{e, m}$ is a simplification in the Rayleigh approximation, when the size parameter $k_{0} R \ll 1$ (the bead radius is much less than the wavelength). Note that $\alpha_{m}$ does not vanish for larger bead sizes and can be described by the second formula in Eqs. (5).

The time-averaged optical force in the field without an intensity gradient in the propagation direction $z,\{\mathbf{E}(\mathbf{r}), \mathbf{H}(\mathbf{r})\}=$ $\mathrm{e}^{\mathrm{i} \beta k_{0} z}\{\mathbf{E}(x, y), \mathbf{H}(x, y)\}$, is written in the dipole approximation as [2]

$$
F_{z}=\frac{k_{0} \beta}{2}\left(\operatorname{Im}\left(\alpha_{e}\right)|\mathbf{E}|^{2}+\operatorname{Im}\left(\alpha_{m}\right)|\mathbf{H}|^{2}\right)-\frac{k_{0}^{4}}{3} \operatorname{Re}\left[\alpha_{e} \alpha_{m}^{*}\left(\mathbf{E} \times \mathbf{H}^{*}\right)_{z}\right]
$$

where $\mathbf{E}$ and $\mathbf{H}$ are the incident fields at the center of the spherical bead $r=0$, and $\beta=k_{z} / k_{0}$ is the dimensionless longitudinal wavenumber. For Rayleigh particles $\alpha_{m}=0$ and the sign of the force $F_{z}=\left(k_{0} \beta / 2\right) \operatorname{Im}\left(\alpha_{e}\right)|\mathbf{E}|^{2}$ depends on the sign of $\operatorname{Im}\left(\alpha_{e}\right)$. Therefore, passive beads are always pushed and gain spheres are always pulled by the incident light.

Polarizabilities (5) and the optical force (6) in the dipole approximation can be used quite solidly for the size parameters of the sphere $k_{0} R \sim 1$. In Fig. 1 one observes the results for anisotropic spheres in the field of a nonparaxial Bessel beam characterized by the order number $m$, cone angle $\alpha$ [see the inset in Fig. 1(a)], and amplitudes of the TE- and TM-polarized Bessel beams $c_{1}$ and $c_{2}$ (see the details of the definition of nonparaxial Bessel beams in $[4,8])$. The dipole approximation accurately predicts the sign of the optical force $\left(F_{z}<0\right.$ for pulling) as shown in Fig. 1(a).

It turns out that the backward or pulling optical force $\left(F_{z}<0\right)$ appears only for $\varepsilon_{t}^{\prime}>0$. Therefore, pulling is realized only for one type of HMMs, namely, for $\varepsilon_{t}^{\prime}>0$ and $\varepsilon_{r}^{\prime}<0$. There is a range of $\varepsilon_{t}^{\prime}$ near 8 where the force is pulling for any $\varepsilon_{r}^{\prime}$, as seen in Fig. 1(a). The maximum negative force $\left|F_{z}\right|$ does not correspond to the isotropic medium case $\varepsilon_{r}=\varepsilon_{t}$, but is shifted towards larger values of $\varepsilon_{r}^{\prime}$. Thus, the particle anisotropy is indeed found to enhance the pulling force effect. However, it can be seen that the strongest pulling is observed for strongly anisotropic media with $\varepsilon_{r}^{\prime}>\varepsilon_{t}^{\prime}>0$ rather than for HMMs.

Remembering that a composite HMM is constructed as a stack of alternating spherical metallic and dielectric layers, we should be ready to account for significant values of losses. However, Fig. 1(b) demonstrates that large 

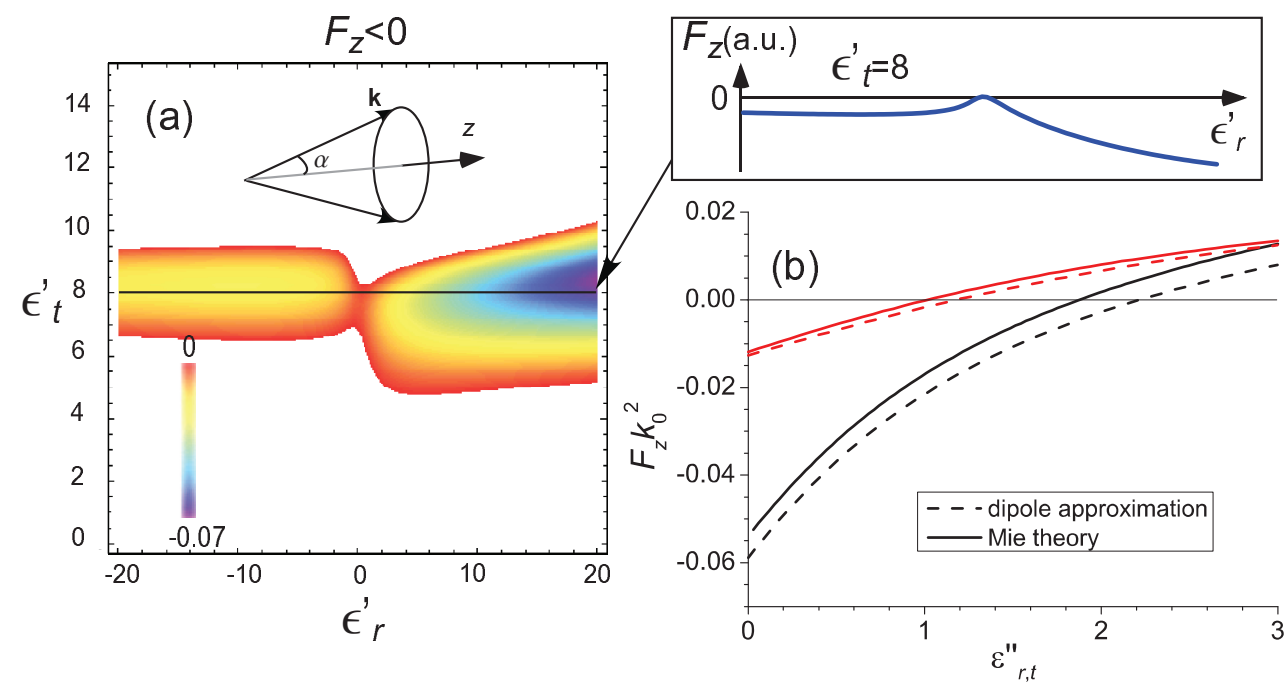

Fig. 1: (a) Pulling optical force $F_{z} k_{0}^{2}<0$ calculated in dipole approximation vs. real parts of the dielectric permittivities $\varepsilon_{r}^{\prime}$ and $\varepsilon_{t}^{\prime}$ (Bessel beam's order $m=1$, loss parameter $\varepsilon_{r, t}^{\prime \prime}=1$ ). The force calculated using the Mie theory (see the curve in the upper right corner) confirms the correctness of the dipole approximation. (b) Optical force $F_{z} k_{0}^{2}$ as a function of the loss parameter $\varepsilon_{r, t}^{\prime \prime}$ (black curves: $m=1, \varepsilon_{r}^{\prime}=-10, \varepsilon_{t}^{\prime}=8$; red curves: $m=-1$, $\varepsilon_{r}^{\prime}=2, \varepsilon_{t}^{\prime}=15$ ). Parameters are: size parameter $k_{0} R=1$, cone angle $\alpha=80^{\circ}$, amplitudes $c_{1}=1$ and $c_{2}=i$.

loss parameter $\varepsilon_{r, t}^{\prime \prime}$ can be tolerated: the optical force remains pulling until $\varepsilon_{r, t}^{\prime \prime} \approx 2$ for the Bessel beam order $m=1$ and until $\varepsilon_{r, t}^{\prime \prime} \approx 1$ for $m=-1$. We see that the optical force depends on $\varepsilon_{r}^{\prime \prime}$ rater weakly, so the pulling effect is not destroyed for significant $\varepsilon_{r}^{\prime \prime}$.

\section{CONCLUSION}

We have analyzed the fields in the vicinity of the center of anisotropic spherical particles and discussed the criterion for choosing the physically appropriate solution. We have revealed that only one type of HMM $\left(\varepsilon_{t}^{\prime}>0\right.$ and $\varepsilon_{r}^{\prime}<0$ ) can be used for making microspheres that are pulled by gradientless beams, and that pulling is preserved even if material losses are significant.

\section{ACKNOWLEDGEMENT}

Support of the Basic Research Foundation of Belarus (grant F13M-014) is acknowledged.

\section{REFERENCES}

[1] C.F. Bohren and D.R. Huffman, Absorption and Scattering of Light by Small Particles, New York: JohnWiley, 1998.

[2] M. Nieto-Vesperinas, J.J. Saenz, R. Gomez-Medina, and L. Chantada, "Optical forces on small magnetodielectric particles," Opt. Express, vol. 18, p. 11428-11443, 2010.

[3] J. Chen, J. Ng, Z. Lin, and C.T. Chan, "Optical pulling force," Nat. Phot., vol. 5, p. 531-533, 2011.

[4] A. Novitsky, C.W. Qiu, and H. Wang, "Single gradientless light beam drags particles as tractor beams," Phys. Rev. Lett., vol. 107, p. 203601, 2011.

[5] S. Sukhov and A. Dogariu, "Negative nonconservative forces: optical tractor beams for arbitrary objects," Phys. Rev. Lett., vol. 107, p. 203602, 2011.

[6] A. Novitsky, C.W. Qiu, and A. Lavrinenko, "Material-independent and size-independent tractor beams for dipole objects," Phys. Rev. Lett., vol. 109, p. 023902, 2012.

[7] V.P. Drachev, V.A. Podolskiy, and A.V. Kildishev, "Hyperbolic metamaterials: new physics behind a classical problem," Opt. Express, vol. 21, p. 15048-15064, 2013.

[8] A.V. Novitsky and D.V. Novitsky, "Negative propagation of vector Bessel beams," J. Opt. Soc. Am. A, vol. 24, p. 2844 2849, 2007. 